\title{
Traditional and ultrasound physical examinations: a hybrid approach to improve clinical care
}

\author{
Marcus Gomes Bastos ${ }^{1}$ \\ Ana Karine Brandão Novaes ${ }^{2}$ \\ José Muniz Pazeli Jr Pazeli
}

1. Department of Clinical Medicine, Faculty of Medicine of the Federal University of Juiz de Fora, Juiz de Fora, /MG, Brasil 2. Faculty of Medicine of the Federal University of Juiz de Fora, Juiz de Fora, /MG, Brasil 3. Faculty of Medicine of the Barbacena, Barbacena/MG, Brasil

http://dx.doi.org/10.1590/1806-9282.64.05.474

\section{SUMMARY}

Point-of-care ultrasonography, which is performed at the bedside by physicians who are not specialists in imaging, has become possible thanks to recent technological advances that have allowed for a device with greater portability while maintaining image quality. The increasing use of point-of-care ultrasonography in different specialties has made it possible to expand physical examinations, make timely decisions about the patients and allows the performance of safer medical procedures. In this review, three cases from our experience are presented that highlight the use of point-of-care ultrasonography by clinicians. Bedside ultrasonography is a convenient modality used in a clinical setting to aid in early diagnosis of several common conditions. It is suggested that a hybrid approach of physical examination and point-of-care ultrasonography in the everyday clinical practice is an inevitable change of paradigm that is improving quality of care in a variety of clinical settings.

KEYWORDS: Ultrasonography. Physical Examination. Nephrotic Syndrome. Aortic Aneurysm, Abdominal. Pneumothorax.

\section{INTRODUCTION}

Traditional physical examinations, which have been performed practically unchanged over the past 200 years, ${ }^{1}$ contribute only $10-20 \%$ to the final diagnostic process; ${ }^{2,3}$ in addition, clinical signs are not always reliable. ${ }^{4}$ In turn, particularly over the past 25 years, the increasing use of point-of-care ultrasonography (POCUS) has been observed. POCUS means the ultrasonography (US) performed at the bedside by physicians who are not specialists in imaging, aiming to answer simple 'yes' or 'no' binomial questions that allow the physical examination to be enhanced by new clinical information, and guid- ing several medical procedures to be performed in a safer way. ${ }^{5}$ The increasing use of POCUS in various medical specialties is due to the growing need for immediate information that cannot be obtained using a traditional physical examination and the greater portability (currently, palmtop devices and applications that allow the use of smartphones to generate the image are available) and affordability of ultrasound devices. ${ }^{6}$

Although there are recommendations based on published evidence for the use of POCUS, ${ }^{7,8}$ and its use is already recognized by foreign societies

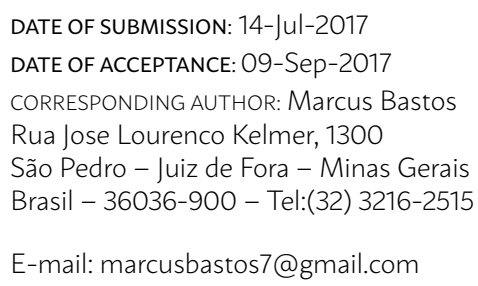


(such as the Society of Critical Care Medicine and the American College of Emergency Physicians), in Brazil, its use is still restricted to a few physicians, particularly those who work in Intensive and/or Emergency Treatment Units. However, it is important to note that a careful clinical history analysis and thorough physical examination will remain the cornerstones of the diagnostic process; as with the stethoscope, reflex hammer and penlight, ultrasound is a tool that, by allowing a "look inside" the patient, should be used not to replace the physical examination but as an extension of it. ${ }^{5}$ Three cases from our experience will be presented and discussed below that highlight how POCUS allows for safer clinical practice of better quality.

\section{CASE REPORT 1:}

A 46-year-old white female patient sought medical care due to lower limb edema, followed by edema around the eyes, which had started approximately two weeks prior. The patient also reported foamy urine and weight gain. Her previous pathological history was unremarkable and she denied the use of continued medication. In the "conventional" physical exam, periorbital edema and edema in the lower limbs up to the root of the thighs (with godet sign) were observed. Her weight was $75 \mathrm{~kg}$ (according to the patient, her "normal" weight is $61 \mathrm{~kg}$ ), and she had a blood pressure of $146 / 84 \mathrm{mmHg}$, non-palpable kidneys and negative bilateral costovertebral angle tenderness. Cardiac-pulmonary auscultation was inexpressive. Abdominal distention, shifting dullness, and bulging flanks were absent. Proteinuria, which was checked with a urine dipstick test, was positive (4+).

Based on the clinical history and findings of the physical examination, particularly for the presence of proteinuria in the urine dipstick test, the diagnosis of nephrotic syndrome was made, and percutaneous renal biopsy was considered for etiological confirmation. However, while discussing the case, the following questions were raised, and POCUS was performed to answer them: 1 . Given the considerable weight gain of the patient, is there fluid in the peritoneal cavity, pleural or pericardial space? 2. Do the kidneys have typical anatomical characteristics that could allow the histologic diagnosis of the underline glomerular disease?

\section{Case Discussion}

Regarding the first question, it is important to note that the use of POCUS in the identification of free intraperitoneal fluid (FIPF) has been recognized since the mid-1980s. ${ }^{9}$ A study using cadavers as a model showed that US allows the physician to visualize up to $100 \mathrm{~mL}$ of FIPF. ${ }^{10}$ However, the use of the US in the evaluation of FIPF gained considerable momentum from research performed on patients with abdominal trauma, leading to the Focused Assessment with Sonography in Trauma (FAST) protocol. In the upper right quadrant window of the FAST (which encompasses Morison pouch, the lower pole of the kidney and the space between the diaphragm and the liver), US can identify volumes of liquid on the order of 500-600 mL. ${ }^{11}$ In the upper left quadrant and suprapubic windows, US has also been used by clinicians in the identification of ascites. The use of POCUS in the diagnosis of ascites was consolidated by the fact that the diagnosis based on the physical examination shows a sensitivity of only $70-80 \%$ in the presence of large volumes of FIPF. ${ }^{12,13}$

Technically, the search for ascites by POCUS is relatively easy and rapid using a low-frequency, high-wavelength probe (convex and/or cardiac). After adjusting for image gain and depth, the FIPF search can be initiated in the upper right quadrant window, followed by the upper left quadrant and suprapubic regions, with the patient in the supine position. In the patient presented above, free liquid (appearing as an anechoic image) was observed in the Morison (Figure 1) and Douglas pouches, confirming the diagnosis of ascites, which was not identified in the physical examination.

FIGURE 1. Ascites, an abnormal collection of fluid that appears as an anechoic image, observed in Morison's pouch (posterior subhepatic space).

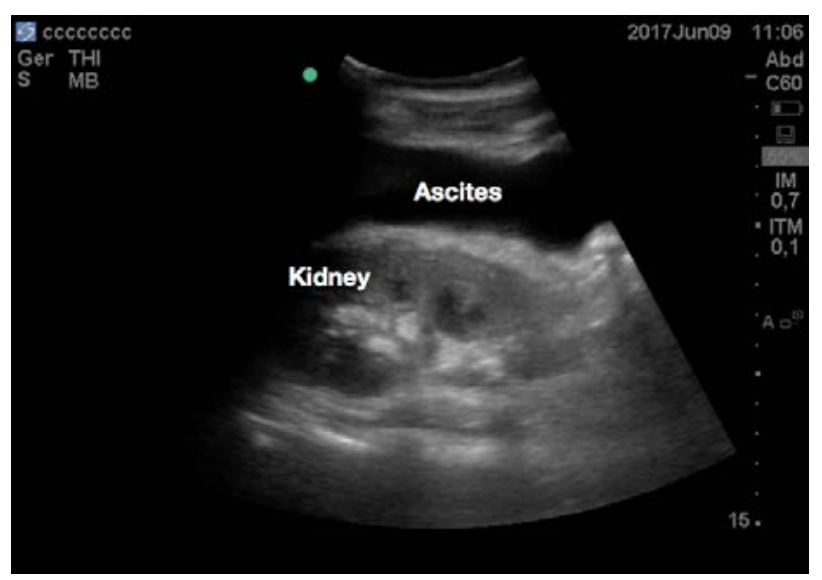

Marcus 
In the thoracic cavity, there are two "enemies" of US: the air inside the lungs and the ribs. Both generate acoustic shadows, which would make it difficult to perform lung ultrasound. Thus, for several years, it was believed that US could not be used to assess pulmonary pathologies. However, in the past two and half decades, US of the lung has gained credibility and is currently one of the primary indications for POCUS, particularly in emergency and intensive care units. An important recommendation of lung US is in the diagnosis of pleural effusion, which is established by the replacement of the mirror image of the liver, an artifact generally observed above the diaphragm, by an anechoic image generated by pleural fluid accumulation. Additionally, pleural effusion can also be identified by the observation of vertebral bodies above the diaphragm, a finding usually not seen due to pulmonary interference, which results from the replacement of lung air by a liquid medium, which is a good conductor of sound. In the present case, pleural effusion was diagnosed examining the pulmonary base between the anterior and posterior axillary lines using a low-frequency convex probe.

Pericardial effusion is a noninflammatory complication of nephrotic syndrome; $;^{14}$ its diagnosis based on a physical examination in mild and moderate cases is challenging. However, transthoracic echocardiography provides a rapid and reliable diagnosis of pericardial effusion and cardiac tamponade..$^{15}$ The diagnosis can be made through the four cardiac windows frequently used in POCUS, that is parasternal long and short axes, apical and subcostal, using

FIGURE 2. Abdominal aortic aneurysm $(6.39 \mathrm{~cm})$ diagnosed during ultrasonography done by the assistant physician during a physical examination of the hypertensive patient.

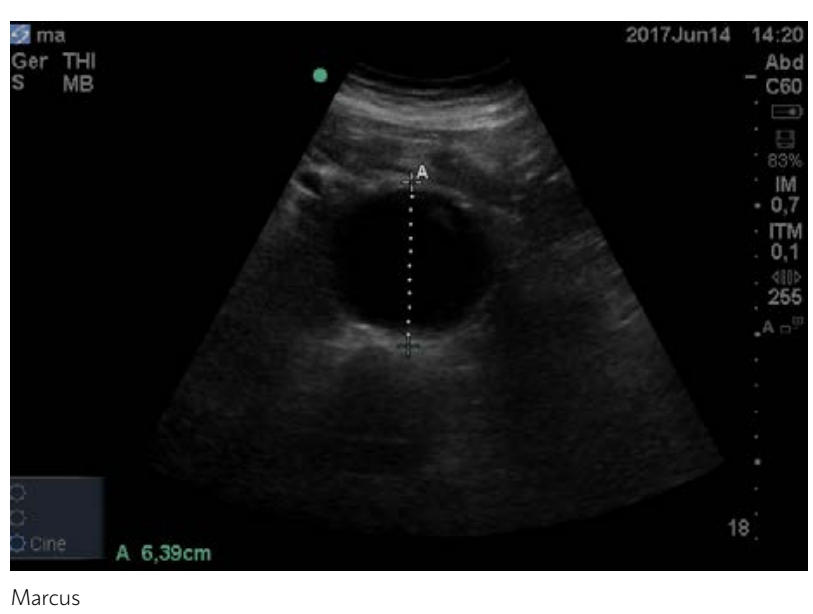

low-frequency probes with small footprints. In the present case, a small pericardial effusion was diagnosed, with no hemodynamic repercussion.

Regarding the second question, if the kidneys presented common anatomical characteristics, POCUS may be fundamental, considering the great difficulty of obtaining renal access through a traditional physical examination. First, because of the decision to perform a renal biopsy, knowledge of whether the patient had both native kidneys was important. Second, since the patient did not present any documentation of previous renal function, it was essential to determine whether her kidneys had normal echotexture and size, as well as corticomedullary differentiation, characteristics that suggest kidneys with proper functionality. ${ }^{16}$ These are important information and indicate that the renal tissue to be obtained is preserved enough to allow the histological diagnosis of the glomerular lesion causing the nephrotic syndrome. The POCUS allowed us to determine that the patient had two kidneys with characteristics of functional preservation, indicating that a renal biopsy was fundamental for characterizing the underlying glomerulonephritis.

\section{CASE REPORT 2:}

A 73-year-old black male patient was seen in the outpatient clinic for blood pressure control. He reported being hypertensive for approximately 26 years and was completely asymptomatic. He had a smoking history that began at 15 years of age (roughly a pack of cigarettes/day), denied having had any previous surgeries, and mentioned that the laboratory evaluation (blood and urine) performed about six months prior was normal. He reported being in regular use of the prescribed antihypertensive medication. During the physical examination, the patient was well oriented, in good general condition, afebrile and with blood pressure of 150/88 mmHg. Pulmonary examination revealed no abnormalities. In the cardiovascular evaluation, cardiac auscultation was interpreted as usual; however, the ankle-brachial in$\operatorname{dex}(\mathrm{ABI})$ showed values lower than 0.9 bilaterally. In the abdomen, inspection, palpation and percussion were inexpressive. The prostate examination was also normal.

However, because he was an elderly male patient with a long-standing history of smoking, hypertensive and with evidence of cardiovascular impairment 
$(\mathrm{ABI}<0.9)$, the following question was raised: "Is the patient's abdominal aorta normal?"

Abdominal US is the screening examination of choice for abdominal aortic aneurysm (AAA) because it is innocuous, can be done expeditiously and at the bedside, with the additional advantage of having high sensitivity of 95 to $100 \%$ and a specificity of nearly $100 \% .{ }^{17,18}$ With the patient in the supine position, the abdominal aorta is scanned at the level of the iliac artery bifurcation with a convex or cardiac transducer (both low-frequency), since approximately $90 \%$ of AAAs occur inferiorly to the renal arteries. ${ }^{19,20}$ For the identification of the aorta, the vertebral bodies, which are hyperechoic and generate the artifact acoustic shadow, are the anatomical landmark. The aorta is identified to the left of the midline, anterior to the vertebral bodies, whereas the inferior vena cava is seen to the right side. The diameter of the abdominal aorta should preferably be measured on the transverse axis from the outer anterior and posterior walls of the vessel. AAA is considered in adults when the aortic diameter is larger than 1.5 times its normal diameter or if the distal aorta exceeds $3 \mathrm{~cm}$. It is always prudent to scan the aorta transversely and longitudinally and over its entire abdominal length.

\section{Case Discussion}

In patients such as the one presented, examination of the abdominal aorta should always be performed. A segmental dilatation of the aortic wall responsible for considerable morbidity and health care costs. ${ }^{21,22}$ AAAs are relatively common and potentially fatal, ${ }^{23}$ and constitute the 14 th leading cause of death in the United States..$^{24}$ AAAs are usually asymptomatic until they expand or rupture. During the period of its expansion, the AAA can cause sudden, severe, and constant back, flank, abdominal, or groin pain. Eventually, these symptoms may be accompanied by episodes of syncope. Large aneurysms in thin people are easy to detect, however, the accuracy of the clinical examination is tremendously reduced by obese body habitus and small aneurysm size. ${ }^{25}$ The occurrence of shock suggests parietal rupture of the AAA, a clinical condition with a high percentage of mortality.

In the patient under discussion, the POCUS allowed the assistant physician to identify an AAA of $6.39 \mathrm{~cm}$ in diameter (Figure 2). Considering that aneurysm size is one of the strongest predictors of the risk of rupture, that such risk increases markedly at aneurysm diameters greater than $5.5 \mathrm{~cm}$, and that the diameter of the AAA diagnosed in our patient has an estimated annual rupture risk of $10 \%$ to $20 \%,{ }^{26}$ the patient was referred for follow-up with a vascular surgeon.

\section{CASE REPORT 3:}

A 25-year-old woman was admitted to the Emergency Unit with nausea and vomiting for 48 hours. The patient said she had had chronic kidney disease diagnosed for approximately 10 years. In the laboratory evaluation, a glomerular filtration rate of 7 $\mathrm{mL} / \mathrm{min} / 1.73 \mathrm{~m}^{2}$, a urea level of $310 \mathrm{mg} / \mathrm{dL}$ and a potassium level of $6.8 \mathrm{mg} / \mathrm{L}$ were observed. The attending physician requested a nephrological evaluation, and immediate hemodialysis treatment was indicated by the nephrologist. As the patient did not have an arteriovenous access previously placed for hemodialysis, the nephrologist decided on central venous access through the right internal jugular vein (IJV). The procedure was based on traditional landmark-based approach as routinely used, but was difficult to perform. After venipuncture, the patient began with dyspnea, and the diagnosis of pneumothorax was raised.

\section{Case Discussion}

Central venous access is a commonly performed procedure with multiple indications in clinical practice, especially in intensive care units. The traditional technique, based on anatomical landmark, is associated with a high risk of failure rates and complications rates as high as $30 \%$ and $18 \%$, respectively, particularly in the needle insertion stage. ${ }^{27}$ Some factors related to the operator's experience and others related to the patient (i.e., obese body habitus, coagulopathies and urgency of the procedure) justify this rate..$^{28-30}$

The use of US for venous access was first described in 1978, but only for the purpose of marking the skin overlying the IJV. ${ }^{31}$ However, only in the mid-eighties the use of real-time US guidance for IJV cannulation was first described. ${ }^{32}$ Therefore, since 2001, American and European societies have included bedside ultrasonography during vascular access as one of 11 practices with "strength of evidence for supporting more widespread implementation", ${ }^{33,34}$ and in 2008 ultrasonographic guidance for venous 
access was listed as a "core or primary emergency ultrasound application" by the American College of Emergency Physicians. ${ }^{8}$

US-guided central vascular access is performed with the patient in the dorsal decubitus position and, in the case presented, the IJV (vessel preferably chosen in clinical situations similar to that of the patient described) is punctured with the head in a neutral position and the operator manipulates the high-frequency transducer ipsilateral next to the patient, from the head of the bed. The anatomical differentiation of the IJV and the carotid artery occurs through compression maneuvers, since the venous system is more compressible than the arterial system, and/or through the Valsalva maneuver when the venous system becomes engorged. It is worth noting that before performing the vessel puncture, it is important to verify its patency, i.e., the absence of venous thrombosis, an absolute contraindication to the procedure that might be present in patients who have had previous accesses in the same vessel. ${ }^{35}$

An IJV access, in general, carries multiple potential complications, being pneumothorax one of the most serious and frequent. The initial diagnosis of pneumothorax is classically based on the chest $\mathrm{X}$-ray, but this imaging modality exhibits a low sensitivity of $36 \%$ to $48 \%$ in some studies. ${ }^{36}$ The sensitivity of lung POCUS in detecting pneumothorax dramatically exceeds that of an X-ray, ${ }^{37}$ with a sensitivity close to that of computed tomography. ${ }^{38-40}$ With the advantage that it can be carried out repeatedly, US also allows the follow-up of the expansion of the pneumothorax by monitoring the point at which the lung touches the thoracic wall (lung point) and, thus, assists in the decision-making process of thoracic drainage before complete pulmonary collapse occurs. An additional advantage is the unnecessary transfer of the patient to the imaging department, which sometimes is impossible.

In the search for pneumothorax using bedside ultrasonography, low-frequency and high-wavelength probes (convex or cardiac) can be used, although the high-frequency linear transducer is most suitable because it provides images of higher-resolution of the more superficial structures.

The examination should begin with the patient in a supine position, with the transducer' marker facing the patient's head and positioned longitudinally over the third or fourth intercostal space in the mid-clavicular line. The parietal and visceral pleura are identi- fied as a hyperechoic line found at the inferior border of the space between the two ribs, sliding one under the other, a sign called lung (or pleural) sliding. The pleural interface should be scanned in at least three different intercostal spaces, concentrating the examination at the most superior and anterior portion of the chest.

The presence of lung sliding excludes the diagnosis of pneumothorax. However, its absence, although sensitive, is not specific for pneumothorax. Since lung sliding represents movement between the two pleurae, other clinical conditions, such as contralateral selective endobronchial intubation, previous pleurodesis, severe pneumonia and acute respiratory distress syndrome, may be associated with its absence. ${ }^{41,42}$

In situations where lung sliding is difficult to discern, the M-mode (movement mode) of US can be used. In M-mode, data referring to a single vertical slice (appears as a vertical line at the first press of the M-mode button) are displayed as a function of time. In M-mode, structures that are typically "still” (from the skin to the pleura) appear as parallel horizontal lines (or "barcode") above the pleura, and the moving lung is viewed as a granular appearance below the

FIGURE 3. Lung ultrasonography in M-mode showing a barcode image above and below the pleural line, compatible with the diagnosis of pneumothorax.

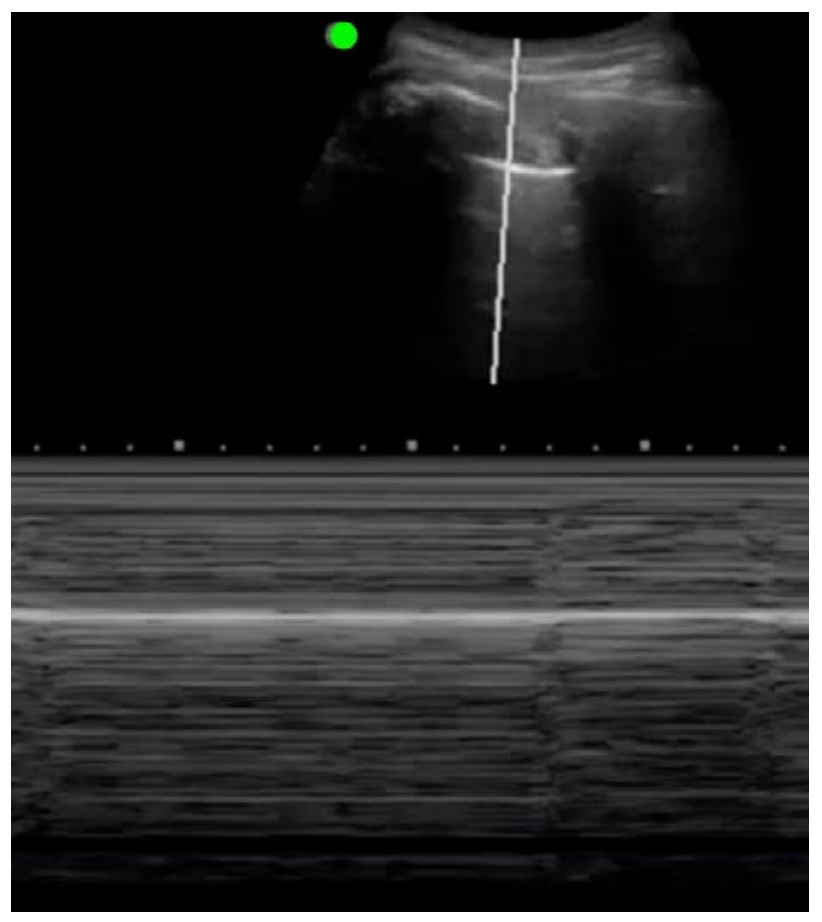

Marcus 
pleura (or "beach sand"), as shown in Figure 4. In the occurrence of pneumothorax, as the lung is "still", the M-mode image appears as a barcode above and below the pleural line (Figure 3). ${ }^{41,43}$

It is important to note that lung US also allows the diagnosis of hemothorax, another complication associated with central venous catheterization, which, unlike pneumothorax, should be investigated at the base of the lung. In addition, POCUS allows the determination of whether the tip of the catheter (particularly double-lumen rigid catheters frequently used in hemodialysis accesses) is correctly positioned at any point in the superior vena cava instead of the right atrium, where it can cause perforation of the cardiac chamber. For this purpose, an injection of saline bolus or previously formed micro-bubbles (by mixing $10 \mathrm{~mL}$ of serum with $1 \mathrm{~mL}$ of air) can be used to determine the position of the tip of the catheter. ${ }^{40}$

In the case under discussion, POCUS of the lung was performed. The absence of pleural (lung) sliding and "barcode" patterns above and below the pleural line in the M-mode observed allowed the diagnosis of pneumothorax due to accidental catheterization of the right IJV.

\section{FINAL COMMENTS}

The practice of medicine is based on the diagnosis and treatment of medical conditions. The three clinical scenarios presented exemplify the importance of incorporating disruptive technologies such as POCUS into medical practice, which can enable the clinician to extend his physical examination, obtain immediate answers to simple questions at the bedside and perform medical procedures that are safer for both the patient and the physician. The current trend is for POCUS to be definitively incorporated into all medical specialties. As is already the case in several medical schools in North America and Europe, and a few in Brazil (ours included), the teaching of US is being incorporated in undergraduate medical education. However, for POCUS to be definitively utilized in everyday medical practice, barriers to its implementation must be supplanted and medical education has to be changed, as proposed by Solomon and Saldana: ${ }^{44}$ "A generation of physicians will need to be trained to view this technology as an extension of their senses, just as many generations have viewed the stethoscope. That development will require the medical education community to embrace and incorporate the technology throughout the curriculum."

\section{REFERENCES}

1. Walker HK. The origins of the history and physical examination. In: Walker HK, Hall WD, Hurst JW, eds. Clinical methods: the history, physical, and laboratory examinations. 3a ed. Boston: Butterworths; 1990.

2. Peterson MC, Holbrook JH, Von Hales D, Smith NL, Staker LV. Contributions of the history, physical examination, and laboratory investigation in making medical diagnoses. West | Med. 1992;156(2):163-5.

3. Sandler G. Costs of unnecessary tests. Br Med J. 1979;2(6181):21-4.

4. Benbassat J, Baumal R. Narrative review: should teaching of the respiratory physical examination be restricted only to signs with proven reliability and validity? J Gen Intern Med. 2010;25(8):865-72.

5. Moore CL, Copel JA. Point-of-care ultrasonography. N Engl J Med. 2011;364(8):749-57.

6. Philips Lumify portable ultrasound machine [Internet]. [cited 2017-4-15]. Available from: http://www. lumify.philips.com/web

7. Frankel HL, Kirkpatrick AW, Elbarbary M, Blaivas M, Desai H, Evans D, et al. Guidelines for the appropriate use of bedside general and cardiac ultrasonography in the evaluation of critically ill patients-part I: general ultrasonography. Crit Care Med. 2015;43(11):2479-502.

8. American College of Emergency Physicians. Emergency ultrasound guidelines. Ann Emerg Med. 2009;53(4):550-70.

9. McGahan JP, Anderson MW, Walter JP. Portable real-time sonographic and needle guidance systems for aspiration and drainage. AJR Am | Roentgenol. 1986;147(6):1241-6.
10. Goldberg BB, Goodman GA, Clearfield HR. Evaluation of ascites by ultrasound. Radiology. 1970;96(1):15-22.

11. Branney SW, Wolfe RE, Moore EE, Albert NP, Heinig M, Mestek M, et al. Quantitative sensitivity of ultrasound in detecting free intraperitoneal fluid. J Trauma. 1995;39(2):375-80.

12. Williams JW Jr, Simel DL. The rational clinical examination. Does this patient have ascites? How to divine fluid in the abdomen. JAMA. 1992;267(19):2645-8.

13. Tibbles CD, Porcaro W. Procedural applications of ultrasound. Emerg Med Clin North Am. 2004;22(3):797-815.

14. Mann DL, Zipes DP, Libby P, Bonow RO. Braunwald's heart disease: a textbook of cardiovascular medicine. 10ª ed. Philadelphia: Elsevier Health Sciences; 2015

15. Adler Y, Charron P, Imazio M, Badano L, Barón-Esquivias G, Bogaert J, et al; European Society of Cardiology (ESC). 2015 ESC Guidelines for the diagnosis and management of pericardial diseases: The Task Force for the Diagnosis and Management of Pericardial Diseases of the European Society of Cardiology (ESC) Endorsed by: The European Association for Cardio-Thoracic Surgery (EACTS). Eur Heart J. 2015;36(42):2921-64.

16. O'Neill WC. Renal ultrasonography: a procedure for nephrologists. Am J Kidney Dis. 1997;30(4):597-85.

17. LaRoy LL, Cormier PI, Matalon TA, Patel SK, Turner DA, Silver B. Imaging of abdominal aortic aneurysms. AJR Am | Roentgenol. 1989;152(4):785-92. 
18. U.S. Preventive Services Task Force. Screening for abdominal aortic aneurysm: recommendation statement. Ann Intern Med. 2005;142(3):198-202.

19. Costa M, Robbs JV. Abdominal aneurysms in a black population: clinicopathological study. Br J Surg. 1986;73(7):554-8.

20. Fowl RJ, Blebea J, Stallion A, Marsch JT, Marsch JG, Love M, et al. Prevalence of unsuspected abdominal aortic aneurysms in male veterans. Ann Vasc Surg. 1993;7(2):117-21.

21. Hirsch AT, Haskal ZI, Hertzer NR, Bakal CW, Creager MA, Halperin JL, et al; American Association for Vascular Surgery; Society for Vascular Surgery; Society for Cardiovascular Angiography and Interventions; Society for Vascular Medicine and Biology; Society of Interventional Radiology; ACC/AHA Task Force on Practice Guidelines Writing Committee to Develop Guidelines for the Management of Patients With Peripheral Arterial Disease; American Association of Cardiovascular and Pulmonary Rehabilitation; National Heart, Lung, and Blood Institute; Society for Vascular Nursing; TransAtlantic Inter-Society Consensus; Vascular Disease Foundation. ACC/AHA 2005 Practice Guidelines for the management of patients with peripheral arterial disease (lower extremity, renal, mesenteric, and abdominal aortic): a collaborative report from the American Association for Vascular Surgery/Society for Vascular Surgery, Society for Cardiovascular Angiography and Interventions, Society for Vascular Medicine and Biology, Society of Interventional Radiology, and the ACC/AHA Task Force on Practice Guidelines (Writing Committee to Develop Guidelines for the Management of Patients With Peripheral Arterial Disease): endorsed by the American Association of Cardiovascular and Pulmonary Rehabilitation; National Heart, Lung, and Blood Institute; Society for Vascular Nursing; Trans-Atlantic Inter-Society Consensus; and Vascular Disease Foundation. Circulation. 2006;113(1):e463-654.

22. Ouriel K, Green RM, Donayre C, Shortell CK, Elliott J, DeWeese JA. An evaluation of new methods of expressing aortic aneurysm size: relationship to rupture. J Vasc Surg. 1992;15(1):12-8.

23. Projeto Diretrizes, Sociedade Brasileira de Angiologia e de Cirurgia vascular (BR). Aneurisma de aorta abdominal: diagnóstico e tratamento. 2015 [cited 2017-5-8] Available from: http://www.sbacv.org.br/institucional/diretrizes-sbacv

24. Silverberg E, Boring CC, Squires TS. Cancer statistics, 1990. CA Cancer Clin. 1990;40(1):9-26.

25. Chervu A, Clagett GP, Valentine RI, Myers SI, Rossi PI. Role of physical examination in detection of abdominal aortic aneurysms. Surgery. 1995;117(4):454-7.

26. Brewster DC, Cronenwett JL, Hallett JW Jr, Johnston KW, Krupski WC Matsuma IS, Joint Council of the American Association for Vascular Surgery and Society for Vascular Surgery. Guidelines for the treatment of abdominal aortic aneurysms. Report of a subcommittee of the Joint Counci of the American Association for Vascular Surgery and Society for Vascular Surgery. J Vasc Surg. 2003;37(5):1106-17.

27. Sznajder II, Zveibil FR, Bitterman H, Weiner P, Bursztein S. Central vein catheterization. Failure and complication rates by three percutaneous approaches. Arch Intern Med. 1986;146(2):259-61.

28. Bhutta ST, Culp WC. Evaluation and management of central venous access complications. Tech Vasc Interv Radiol. 2011;14(4):217-24.

29. Feller-Kopman D. Ultrasound-guided internal jugular access: a proposed standardized approach and implications for training and practice. Chest. 2007;132(1):302-9.

30. Dietrich CF, Horn R, Morf S, Chiorean L, Dong Y, Cui XW, et al. Ultrasound-guided central vascular interventions, comments on the European Federation of Societies for Ultrasound in Medicine and Biology guidelines on interventional ultrasound. J Thorac Dis. 2016;8(9):E851-68.

31. Ullman II, Stoelting RK. Internal jugular vein location with the ultrasound Doppler blood flow detector. Anesth Analg. 1978;57(1):118.

32. Yonei A, Nonoue T, Sari A. Real-time ultrasonic guidance for percutaneous puncture of the internal jugular vein. Anesthesiology. 1986;64(6):830-1.

33. Dexheimer Neto FLD, Teixeira C, Oliveira RP. Acesso venoso central guiado por ultrassom: qual a evidência? Rev Bras Ter Intensiva. 2011;23(2):217-21.

34. Brass P, Hellmich M, Kolodziej L, Schick G, Smith AF. Ultrasound guidance versus anatomical landmarks for subclavian or femoral vein catheterization. Cochrane Database Syst Rev. 2015;1:CD011447.

35. Graham AS, Ozment C, Tegtmeyer K, Lai S, Braner DA. Videos in clinical medicine. Central venous catheterization. N Engl | Med. 2007;356(21):e21.

36. Soult MC, Weireter LI, Britt RC, Collins IN, Novosel TI, Reed SF, et al. Can routine trauma bay chest $x$-ray be bypassed with an extended focused assessment with sonography for trauma examination? Am Surg. 2015;81(4):336-40.

37. Rowan KR, Kirkpatrick AW, Liu D, Forkheim KE, Mayo JR, Nicolaou S. Traumatic pneumothorax detection with thoracic US: correlation with chest radiography and CT: initial experience. Radiology. 2002;225(1):210-4

38. Blaivas $M$, Lyon M, Duggal S. A prospective comparison of supine chest radiography and bedside ultrasound for the diagnosis of traumatic pneumothorax. Acad Emerg Med. 2005;12(9):844-9.

39. Cortellaro F, Colombo S, Coen D, Duca PG. Pulmonary ultrasound is an accurate diagnostic tool for the diagnosis of pneumonia in the emergency department. Emerg Med J. 2012;29(1):19-23.

40. Vezzani A, Manca T, Vercelli A, Braghieri A, Magnacacallo A. Ultrasonography as a guide during vascular access procedures and in the diagnosis of complications. J Ultrasound. 2013;16(4):161-70.

41. Lichtenstein DA, Menu Y. A bedside ultrasound sign ruling out pneumothorax in the critically ill. Lung sliding. Chest. 1995:108(5):1345-8.

42. Gargani L, Volpicelli G. How I do it: lung ultrasound. Cardiovasc Ultrasound. 2014;12:25.

43. Irwin Z, Cook JO. Advances in point-of-care thoracic ultrasound. Emerg Med Clin North Am. 2016;34(1):151-7.

44. Solomon SD, Saldana F. Point-of-care ultrasound in medical education: stop listening and look. New Engl | Med. 2014;370(12):1083-5. 